\title{
О ВЛИЯНИИ ВЫЧИСЛИТЕЛЬНЫХ ПОГРЕШНОСТЕИ НА РЕШЕНИЕ ОБРАТНОЙ ЗАДАЧИ АТМОСФЕРНОЙ ОПТИКИ ПО ОПРЕДЕЛЕНИЮ ПОГЛОЩАЮЩИХ КОМПОНЕНТ АТМОСФЕРЫ
}

\author{
(Представил Г. Кузмин)
}

В настоящей работе исследуется оценка погрешности, вызванной упрощением расчета многократно рассеянной солнечной радиации при решении обратной задачи по определению поглощающих компонент атмосферы. Обратная задача определения распределения концентрации водяного пара для сферической модели атмосферы решается с учетом и без учета многократно рассеянного излучения. Проводится сравнение вариантов решения.

\section{1. Модель атмосферы. Постановка задачи}

Рассмотрим следующую модель атмосферы. На сферическую поверхность падает параллельный поток солнечного излучения. Атмосфера разбита на $n$ слоев, в каждом из которых концентрация $\eta_{i}$ поглощающей компоненты атмосферы взята постоянной $(i=1,2, \ldots, n)$. В точке $S$ вне атмосферы находится спутник, измеряющий интенсивность солнечного излучения, рассеянного атмосферой и приходящего в точку $S$ по $l$ различным направлениям. Значение интенсивности в направлении c номером $k$, которое соответствует параметру поглощения $\eta=$ $=\left(\eta_{1}, \eta_{2}, \ldots, \eta_{n}\right)$, обозначим через $J_{k}\left(\eta_{1}, \eta_{2}, \ldots, \eta_{n}\right)$ и будем рассматривать как функцию параметра $\eta$.

Пусть нам известны значения $I_{k}$ интенсивностей солнечного излучения, приходящего в точку $S$ по направлению с номером $k$. Рассмотрим обратную задачу определения параметра $\eta$ по известным значениям $I_{h}$ интенсивностей $\left[{ }^{1}\right]$, т. е. рассмотрим систему нелинейных уравнений

$$
J_{k}\left(\eta_{1}, \eta_{2}, \ldots, \eta_{n}\right)=I_{k}, \quad k=1,2, \ldots, l,
$$

или в векторной форме

$$
J(\eta)=I .
$$

Представим вектор-функцию $J(\eta)$ в виде

$$
J(\eta)=h(\eta)+g(\eta)
$$

где $h(\eta)$ - вектор значений интенсивностей однократно рассеянного и 
приходящего в точку $S$ солнечного излучения, а $g(\eta)$ - вектор, учитываюий все остальное излучение

$$
g(\eta)=J(\eta)-h(\eta)
$$

При решении задачи (1) нам нужно располагать значениями векторфункции $J(\eta)$-в некоторых точках $\eta \in R^{n}$. Наибольшие затруднения $[2,3]$ вызывает расчет функции $g(\eta)$ в выражении (2). Поэтому в целях некоторого упрощения методики расчета ее заменяют некоторой функцией $\tilde{g}(\eta)$. В частности, если нам известно, что значение $g(\eta)$ интенсивности многократно рассеянного и приходящего в точку $S$ солнечного излучения мало по сравнению с $h(\eta)$, то можно положить $\tilde{g}(\eta):=0$.

Запишем уравнение (1) в виде

$$
h(\eta)-u(\eta)=0,
$$

где $u(\eta)=I-\tilde{g}(\eta)$. Вместо уравнения (3) рассмотрим уравнение

$$
h(\eta)-\tilde{u}(\eta)=0
$$

где $\tilde{u}(\eta)=I-\tilde{g}(\eta)$.

В настоящей работе изучается «отклонение» решения $\tilde{\eta}$ задачи (4) от истинного решения (3). Численно сравнивается решение задачи (3), где $\eta$ есть распределение концентрации водяного пара, и решение задачи $(4)$, где $\widetilde{g}(\eta)=0$. Расчеты проведены для модели атмосферы $\left[{ }^{4}\right]$.

\section{2. Математическая постановка задачи}

Пусть на множестве

$$
\left[a^{0}, b^{0}\right]=\left\{x \in R^{n} ; a_{j}^{0} \leqslant x_{j} \leqslant b_{j}^{0}, j=1,2, \ldots, n\right\}
$$

заданы непрерывные функции

$$
h, g, \tilde{g}: R^{n} \rightarrow R^{l} .
$$

Введем частичную упорядоченность на $\left[a^{0}, b^{0}\right]$ при помощи отношения $\left[{ }^{5}\right]$

$$
x \leqslant y \Leftrightarrow y-x \in K=\left\{z \in R^{n} ; z_{j} \geqslant 0, j=1,2, \ldots, n\right\} .
$$

Предположим, что функции $h, g, \tilde{g}$ монотонно убывают, т. е. для любых $v, u \in R^{n}$ таких, что $v \leqslant u$, следует

$$
h(u) \leqslant h(v), \quad g(u) \leqslant g(v), \quad \tilde{g}(u) \leqslant \tilde{g}(v) .
$$

Для каждого вектора $v \in R^{n}$ с координатами $v_{j}$ определим вектор $|v|$ с координатами $\left|v_{j}\right|$. Предположим, что для любого $\eta \in\left[a^{0}, b^{0}\right]$ имеет место неравенство

$$
|g(\eta)-\tilde{g}(\eta)| \leqslant \varepsilon
$$

где $\varepsilon$ - вектор, характеризующий погрешность $\tilde{g}(\eta)$.

Рассмотрим уравнения

$$
\begin{aligned}
& z(\eta) \equiv h(\eta)-u(\eta)=0, \\
& \tilde{z}(\eta) \equiv h(\eta)-\tilde{u}(\eta)=0,
\end{aligned}
$$

где $u(\eta)=I-g(\eta)$, а $\tilde{u}(\eta)=I-\widetilde{g}(\eta)$. Заметим, что в уравнениях 
(6) и (7) функции $u(\eta)$ и $\widetilde{u}(\eta)$ монотонно возрастают (т. е. функции $-u(\eta)$ и - $\widetilde{u}(\eta)$ монотонно убывают).

Предположим, что уравнения (6) и (7) имеют решения, принадлежащие множеству $\left[a^{0}, b^{0}\right]$. Обозначим эти решения через $\eta^{*}$ и $\tilde{\eta}$ соответственно и исследуем величину $\Delta=\left|\eta^{*}-\tilde{\eta}\right|$.

\section{3. Сходимость приближенных решений}

Т еорем а 1. Пусть задача (6) имеет единственное решение $\eta^{*} \in$ $\in\left[a^{0}, b^{0}\right]$. Тогда, если существует решение $\eta_{\varepsilon}$ задачи (7) такое, что

$$
\eta_{\varepsilon} \in\left[a^{0}, b^{0}\right] \forall\|\varepsilon\|_{1} \leqslant \varepsilon_{0},
$$

имеет место сходимость

$$
\left\|\eta_{\varepsilon}-\tilde{\eta}\right\|_{2} \rightarrow 0 \quad n p u \quad\|\varepsilon\|_{1} \rightarrow 0,
$$

где $\varepsilon_{0}$ - некоторое число, а $\|\cdot\|_{1} u\|\cdot\|_{2}-$ некоторые нормы $R^{l} u R^{n}$ соответственно.

Д ок а з ат ел ь с в о. Из соотношения

$$
\left|h\left(\eta_{\varepsilon}\right)-u\left(\eta_{\varepsilon}\right)\right|=\left|\tilde{u}\left(\eta_{\varepsilon}\right)-u\left(\eta_{\varepsilon}\right)\right| \leqslant \varepsilon
$$

следует неравенство

$$
\left\|h\left(\eta_{\varepsilon}\right)-u\left(\eta_{\varepsilon}\right)\right\|_{1} \leqslant\|\varepsilon\|_{1}
$$

Для всех $\varepsilon$ таких, что $\|\varepsilon\| \leqslant \varepsilon_{0}$, множество решений $\eta_{\varepsilon}$ уравнения (7) компактно. Пусть $\eta_{\varepsilon_{n}}$ - некоторая сходящаяся подпоследовательность. Тогда, учитывая (8), получим, что $\eta_{\varepsilon_{n}} \rightarrow \eta^{*}$. Отсюда, ввиду единственности решения уравнения (8), вытекает, что компактное множество решений $\eta_{\varepsilon}$ уравнения (7) при $\|\varepsilon\| \leqslant \varepsilon_{0}$ имеет единственную предельную точку $\eta^{*}$. Следовательно,

$$
\left\|\eta_{\varepsilon}-\eta^{*}\right\|_{2} \rightarrow 0 \text { при }\|\varepsilon\|_{1} \rightarrow 0 .
$$

Теорема доказана.

Рассмотрим множество

$$
S(\tilde{\eta})=\left\{x \in\left[a^{0}, b^{0}\right] ;|h(x)-\tilde{u}(\tilde{\eta})| \leqslant \delta(\varepsilon)\right\},
$$

где

$$
\delta(\varepsilon)=\left|\tilde{u}(\tilde{\eta})-u\left(\eta^{*}\right)\right|
$$

Из равенства

$$
\left|h\left(\eta^{*}\right)-\tilde{u}(\tilde{\eta})\right|=\left|\tilde{u}(\tilde{\eta})-u\left(\eta^{*}\right)\right|=\delta(\varepsilon)
$$

следует, что решение $\eta^{*}$ задачи (6) принадлежит множеству $S(\tilde{\eta})$.

\section{4. Оценка множества $S(\tilde{\eta})$}

Приведем алгоритм оценки множества $S(\tilde{\eta})$ для случая $l=n$.

Рассмотрим последовательности векторов $\left(a^{p}\right)_{p \in N}$ и $\left(b^{p}\right)_{p \in N}$, координаты которых $a_{j}^{p}$ и $b_{j}^{p}$ удовлетворяют уравнениям 


$$
\begin{gathered}
h_{j}\left(b_{1}^{p}, \ldots, b_{j-1}^{p}, a_{j}^{p}, b_{j+1}^{p-1}, \ldots, b_{n}^{p-1}\right)=\xi_{j}, \\
h_{j}\left(a_{1}^{p}, \ldots, a_{j-1}^{p}, b_{j}^{p}, a_{j+1}^{p-1}, \ldots, a_{n}^{p-1}\right)=\xi_{j}, \quad j=1,2, \ldots, n,
\end{gathered}
$$

где $h_{j}\left(x_{1}, x_{2}, \ldots, x_{n}\right)$ означает $j$-ю координату вектора $h(x)$, а $\xi_{j}$ и $\xi_{j}$ есть $j$-я координата векторов $\tilde{u}(\tilde{\eta})+\delta(\varepsilon)$ и $\tilde{u}(\tilde{\eta})-\delta(\varepsilon)$ соответственно.

Обозначим

$$
\begin{aligned}
c_{j}^{p}(t) & =h_{j}\left(b_{1}^{p}, \ldots, b_{j-1}^{p}, t, b_{j+1}^{p-1}, \ldots, b_{n}^{p-1}\right), \\
d_{j}^{p}(t) & =h_{j}\left(a_{1}^{p}, \ldots, a_{j-1}^{p}, t, a_{j+1}^{p-1}, \ldots, a_{n}^{p-1}\right) .
\end{aligned}
$$

В силу предположения относительно функции $h(x)$ функции $c_{j}^{p}(t)$ и $d_{j}^{p}(t)$ являются непрерывными и монотонно убывающими на $\left[a_{j}{ }^{0}, b_{j}{ }^{0}\right]$.

Л ем м а 1. Пусть существуют первые члены $a^{1}, b^{1} \in\left[a^{0}, b^{0}\right]$ последовательности (9). Тогда

$$
S(\tilde{\eta}) \subseteq\left[a^{1}, b^{1}\right] \subseteq\left[a^{0}, b^{0}\right] .
$$

Доказ а тельство проведем методом математической индукции. Покажем, что

$$
\forall \alpha \in S(\tilde{\eta}) \Rightarrow \alpha_{1} \in\left[a_{1}^{1}, b_{1}^{1}\right] \subseteq\left[a_{1}^{0}, b_{1}^{0}\right]
$$

Пусть $\alpha \in S(\tilde{\eta})$. Тогда, по определению $S(\tilde{\eta})$, имеем

$$
\begin{aligned}
& \xi_{1} \leqslant h_{1}\left(\alpha_{1}, \alpha_{2}, \ldots, \alpha_{n}\right) \leqslant h_{1}\left(\alpha_{1}, a_{2}^{0}, \ldots, a_{n}^{0}\right)=d_{1}^{1}\left(\alpha_{1}\right), \\
& \xi_{1} \geqslant h_{1}\left(\alpha_{1}, \alpha_{2}, \ldots, \alpha_{n}\right) \geqslant h_{1}\left(\alpha_{1}, b_{2}^{0}, \ldots, b_{n}^{0}\right)=c_{1}^{1}\left(\alpha_{1}\right) .
\end{aligned}
$$

Отсюда

$$
\alpha_{1} \in\left\{t \in R ; d_{1}^{1}(t) \geqslant \xi_{1}, c_{1}^{1}(t) \leqslant \xi_{1}\right\} .
$$

Учитывая, что $d_{1}{ }^{1}(t) \geqslant c_{1}{ }^{1}(t)$ при $t \in\left[a_{1}{ }^{0}, b_{1}{ }^{0}\right]$ и $\zeta_{1} \leqslant \xi_{1}$, получим

$$
\left\{t \in R ; d_{1}^{1}(t) \geqslant \zeta_{1}, c_{1}^{1}(t) \leqslant \xi_{1}\right\}=\left[a_{1}^{1}, b_{1}^{1}\right],
$$

где $a_{1}{ }^{1}$ и $b_{1}{ }^{1}$ удовлетворяют уравнениям

$$
d_{1}^{1}\left(b_{1}^{1}\right)=\xi_{1}, \quad c_{1}^{1}\left(a_{1}^{1}\right)=\xi_{1} .
$$

Пусть утверждение доказано для $(j-1)$ координат. Тогда

$$
\begin{aligned}
& \zeta_{j} \leqslant h_{j}\left(\alpha_{1}, \alpha_{2}, \ldots, \alpha_{n}\right) \leqslant h_{j}\left(a_{1}^{1}, \ldots, a_{j-1}^{1}, \alpha_{j}, a_{j+1}^{0}, \ldots, a_{n}^{0}\right)=d_{j}^{1}\left(\alpha_{j}\right), \\
& \xi_{j} \geqslant h_{j}\left(\alpha_{1}, \alpha_{2}, \ldots, \alpha_{n}\right) \geqslant h_{j}\left(b_{1}^{1}, \ldots, b_{j-1}^{1}, \alpha_{j}, b_{j+1}^{0}, \ldots, b_{n}^{0}\right)=c_{j}^{1}\left(\alpha_{j}\right) .
\end{aligned}
$$

Отсюда

$$
\alpha_{j} \in\left\{t \in R ; d_{j}^{1}(t) \geqslant \xi_{j}, c_{j}^{1-}(t) \leqslant \xi_{j}\right\}=\left[a_{j}^{1}, b_{j}^{1}\right],
$$

где $a_{j}{ }^{1}$ и $b_{j}{ }^{1}$ удовлетворяют уравнениям

$$
d_{j}^{1}(t)=\xi_{j}, \quad c_{j}^{1}(t)=\xi_{j} .
$$

Лемма доказана. 
Л ем м а 2. Пусть $c_{j}^{p}(t) \leqslant d_{j}^{p}(t) u a^{p-1} \leqslant b^{p-1}$. Тогда неравенства

$$
c_{j}^{p}\left(a_{j}^{p-1}\right) \geqslant \xi_{j}, \quad d_{j}^{p}\left(b_{j}^{p-1}\right) \leqslant \xi_{j}, \quad j=1,2, \ldots, n,
$$

являются необходимыми и достаточными условиями существования решений $a_{j}^{p}, b_{j}^{p} \in\left[a_{j}^{p-1}, b_{j}^{p-1}\right]$ уравнений

$$
c_{j}^{p}(t)=\xi_{j}, \quad d_{j}^{p}(t)=\xi_{j}, \quad j=1,2, \ldots, n .
$$

Доказательство. Ввиду монотонности функций $c_{j}^{p}(t)$ и $d_{j}^{p}(t)$ необходимым и достаточным условием существования в $\left[a_{j}^{p-1}, b_{j}^{p-1}\right]$ решений уравнения (12) есть

$$
c_{j}^{p}\left(a_{j}^{p-1}\right) \geqslant \xi_{j}, \quad c_{j}^{p}\left(b_{j}^{p-1}\right) \leqslant \xi_{j}, \quad d_{j}^{p}\left(a_{j}^{p-1}\right) \geqslant \xi_{j}, \quad d_{j}^{p}\left(b_{j}^{p-1}\right) \leqslant \xi_{j} .
$$

Учитывая, что $c_{j}{ }^{p}(t) \leqslant d_{j}{ }^{p}(t)$ и $\zeta_{j} \leqslant \xi_{j}$, получим, что неравенства (13) эквивалентны неравенствам (11).

Лемма доказана.

Т еорем а 2. Если существуют первые члены $a^{1}, b^{1} \in\left[a^{0}, b^{0}\right]$ в последовательности (10), то существуют р-е члены $a^{p} u b^{p}$ в последовательности (9), причем

$$
S(\tilde{\eta}) \subseteq\left[a^{p}, b^{p}\right] \subseteq \ldots \subseteq\left[a^{1}, b^{1}\right] \subseteq\left[a^{0}, b^{0}\right] .
$$

Д ок а з тельство проведем методом математической индукции. Для $p=1$ утверждение имеет место в силу леммы 1. Предположим, что утверждение имеет место и для $(p-1)$. Покажем, что тогда $a_{j}{ }^{p}$ и $b_{j}{ }^{p}$ существуют и

$$
\forall \alpha \in S(\tilde{\eta}) \Rightarrow \alpha_{j} \in\left[a_{j}^{p}, b_{j}^{p}\right] \subseteq\left[a_{j}^{p-1}, b_{j}^{p-1}\right] .
$$

Для доказательства последнего утверждения вновь применим метод математической индукции. Пусть $j=1$. Так как $a^{0} \leqslant a^{1} \leqslant \ldots \leqslant a^{p-1}$ и $b^{0} \geqslant b^{1} \geqslant \ldots \geqslant b^{p-1}$ (по предположению индукции для $(p-1)$-го шага) и функции $c_{j}{ }^{k}(t)$ и $d_{j}^{k}(t)$ убывают, то

$$
\begin{aligned}
& c_{1}^{1}(t) \leqslant c_{1}^{2}(t) \leqslant \ldots \leqslant c_{1}^{p-1}(t) \leqslant c_{1}^{p}(t), \\
& d_{1}^{1}(t) \geqslant d_{1}^{2}(t) \geqslant \ldots \geqslant d_{1}^{p-1}(t) \geqslant d_{1}^{p}(t) .
\end{aligned}
$$

Отсюда

$$
\begin{aligned}
& \xi_{1}=c_{1}^{p-1}\left(a_{1}^{p-1}\right) \leqslant c_{1}^{p}\left(a_{1}^{p-1}\right), \\
& \xi_{1}=d_{1}^{p-1}\left(b_{1}^{p-1}\right) \geqslant d_{1}^{p}\left(b_{1}^{p-1}\right) .
\end{aligned}
$$

Согласно лемме 2, уравнения

$$
c_{1}^{p}(t)=\xi_{1}, \quad d_{1}^{p}(t)=\xi_{1}
$$

имеют решения $a_{1}^{p}, b_{1}{ }^{p} \in\left[a_{1}^{p-1}, b_{1}^{p-1}\right]$. Далее, для любого $\alpha \in S(\tilde{\eta}) \subseteq$ $\subseteq\left[a_{1}^{p-1}, b_{1}^{p-1}\right]$ верно

$$
\begin{aligned}
& \zeta_{1} \leqslant h_{1}\left(\alpha_{1}, \alpha_{2}, \ldots, \alpha_{n}\right) \leqslant h_{1}\left(\alpha_{1}, a_{2}^{p-1}, \ldots, a_{n}^{p-1}\right)=d_{1}^{p}\left(\alpha_{1}\right), \\
& \xi_{1} \geqslant h_{1}\left(\alpha_{1}, \alpha_{2}, \ldots, \alpha_{n}\right) \geqslant h_{1}\left(\alpha_{1}, b_{2}^{p-1}, \ldots, b_{n}^{p-1}\right)=c_{1}^{p}\left(\alpha_{1}\right) .
\end{aligned}
$$




\section{Отсюда}

$$
\alpha_{1} \in\left\{t \in\left[a_{1}^{p-1}, b_{1}^{p-1}\right] ; a_{1}^{p}(t) \geqslant \zeta_{1}, c_{1}^{p}(t) \leqslant \xi_{1}\right\}=\left[a_{1}^{p}, b_{1}^{p}\right] .
$$

Пусть утверждение доказано для $(j-1)$ координат. Тогда

$$
\begin{aligned}
& c_{j}^{1}(t) \leqslant c_{j}^{2}(t) \leqslant \ldots \leqslant c_{j}^{p}(t), \\
& d_{j}^{1}(t) \geqslant d_{j}^{2}(t) \geqslant \ldots \geqslant d_{j}^{p}(t), \\
& \xi_{j}=c_{j}^{p-1}\left(a_{j}^{p-1}\right) \leqslant c_{j}^{p}\left(a_{j}^{p-1}\right), \\
& \zeta_{j}=d_{j}^{p-1}\left(b_{j}^{p-1}\right) \geqslant d_{j}^{p}\left(b_{j}^{p-1}\right), \quad j=1,2, \ldots, n .
\end{aligned}
$$

Отсюда, по лемме 2 , существуют $a_{j}^{p}$ и $b_{j}^{p}$, удовлетворяющие уравнениям

$$
c_{j}^{p}\left(a_{j}^{p}\right)=\xi_{j}, \quad d_{j}^{p}\left(b_{j}^{p}\right)=\zeta_{j} .
$$

Для произвольного $x \in S(\tilde{\eta})$ имеем

$$
\begin{aligned}
& \zeta_{j} \leqslant h_{j}\left(x_{1}, x_{2}, \ldots, x_{n}\right) \leqslant h_{j}\left(a_{j}^{p}, \ldots, a_{j-1}^{p}, x_{j}, a_{j+1}^{p-1}, \ldots, a_{n}^{p-1}\right)=d_{j}^{p}\left(x_{j}\right), \\
& \xi_{j} \geqslant h_{j}\left(x_{1}, x_{2}, \ldots, x_{n}\right) \geqslant h_{j}\left(b_{j}^{p}, \ldots, b_{j-1}^{p}, x_{j}, b_{j+1}^{p-1}, \ldots, b_{n}^{p-1}\right)=c_{j}^{p}\left(x_{j}\right),
\end{aligned}
$$

откуда

$$
x_{j} \in\left\{t \in\left[a_{j}^{p-1}, b_{j}^{p-1}\right] ; d_{j}^{p}(t) \geqslant \zeta_{j}, c_{j}^{p}(t) \leqslant \xi_{j}\right\}=\left[a_{j}^{p}, b_{j}^{p}\right],
$$

что и доказывает теорему.

Из теоремы следует, что

$$
\begin{aligned}
S(\tilde{\eta}) & \subseteq \bigcap_{p=0}^{\infty}\left[a^{p}, b^{p}\right]=\left[a^{*}, b^{*}\right], \\
\Delta & =\left|\tilde{\eta}-\eta^{*}\right| \leqslant b^{*}-a^{*} .
\end{aligned}
$$

\section{5. Численные результаты}

Рассмотрим обратную задачу определения распределения концентрации водяного пара по измеренному из космоса полю яркости атмосферы для модели $\left[{ }^{4}\right]$.

В точку $S$ по направлению $i$ поступает однократно рассеянное излучение интенсивностью

$$
h_{i}\left(\eta_{1}, \eta_{2}, \ldots, \eta_{n}\right)=c_{0} \int_{0}^{T_{t}} W(\gamma) \exp (-(\tau+\varrho(\tau)) P(\eta ; \tau) \mathrm{d} \tau,
$$

где $c_{0}=\pi S-$ солнечная постоянная, $T_{i}$ - оптическая толщина атмосферы в направлении $i, \gamma$ - угол между направлением визирования и солнечным лучом, $\varrho(\tau)$ - оптический путь от Солнца до точки, лежащей на линии визирования и находящейся на оптическом расстоянии $\tau$ от спутника, $W(\gamma)$ - индикатриса рассеяния, нормированная условием $\int W(\gamma) \mathrm{d} \omega=1$, а $P(\eta ; \tau)-$ функция пропускания.

Как показали расчеты $\left[{ }^{4}\right]$, значение $g(\eta)$ интенсивности многократно рассеянного солнечного излучения в модели атмосферы с небольшими 


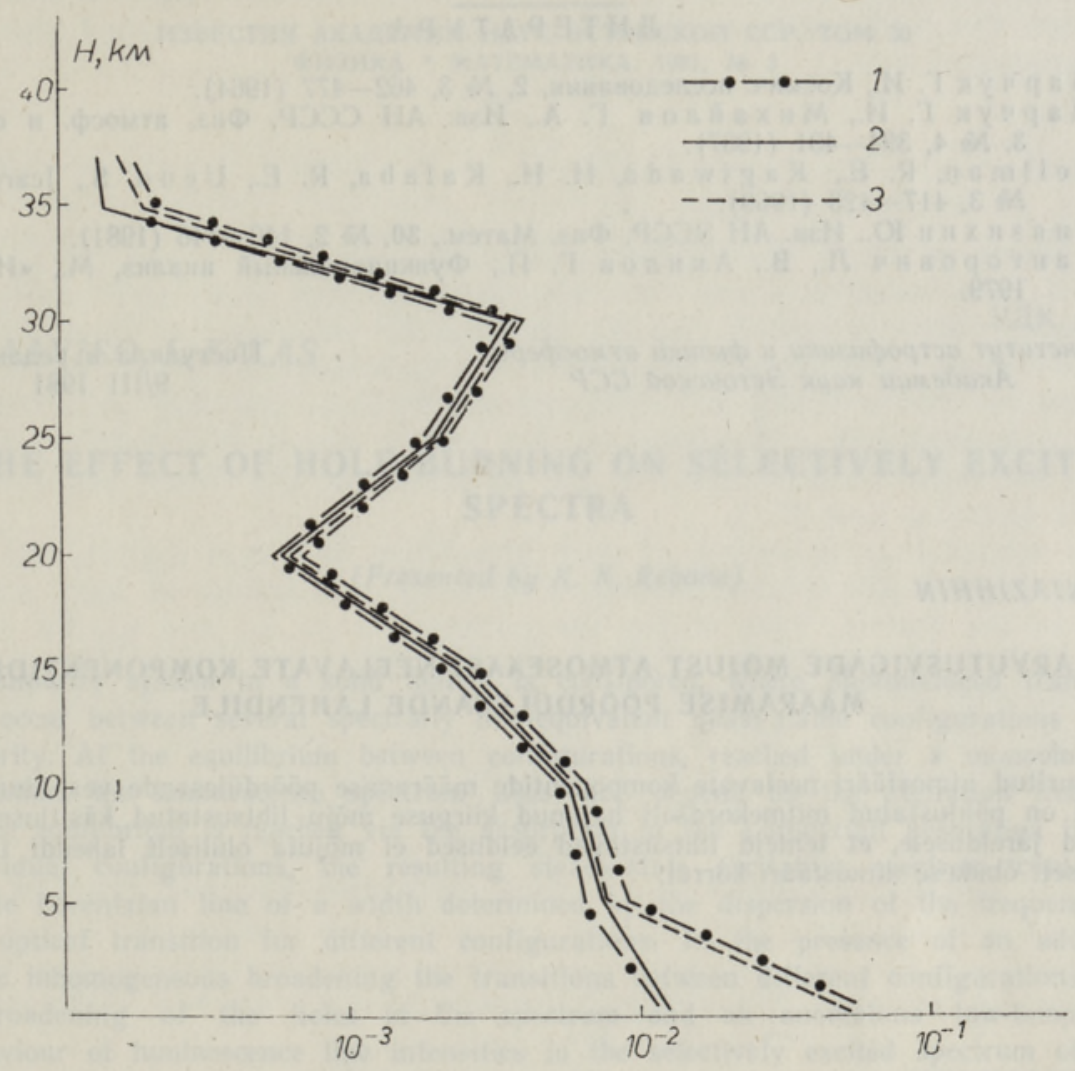

1 - высотный ход распредєления концентрации водяного пара, полученный при решении уравнения (14); 2 - то же при решении уравнения (15);

$$
3 \text { - оценка границ множества } S(\tilde{\eta}) \text {. }
$$

оптическими толщинами мало по сравнению со значением $h(\eta)$ интенсивности однократно рассеянного солнечного излучения. Поэтому положим $\tilde{g}(\eta)=0$ и рассмотрим задачу

$$
h(\eta)=I .
$$

На рисунке показан высотный ход распределения концентрации вадяного пара, полученный при решении уравнения (14) и уравнения

$$
h(\eta)+g(\eta)=I,
$$

а также верхние и нижние границы соответствующего этой задаче множества $S(\tilde{\eta})$, где $\delta(\varepsilon)=\varepsilon$.

Уравнение (14) решали, используя итерационный процесс (9), где $\xi=\xi=I$, а уравнение (15) - методом, разработанным в [ $\left.{ }^{4}\right]$. Как видно из рисунка, при решении рассмотренной обратной задачи для модели атмосферы с малой оптической толщиной многократно рассеянным излучением можно пренебрегать. 
ЛИТЕРА Т У Р А

1. М а р ч ук Г. И., Космич. исследования, 2, № 3, 462-477 (1964).

2. М ар чук Г. И., М их йлов Г. А., Изв. АН СССР, Физ. атмосф. и океана, 3, № 4, 394-401 (1967).

3. Be $11 \mathrm{~m}$ a n, R. E., K a g i w a d a, H. H., Kal aba, R. E., U e n o, S., Icarus, 11, № 3, 417-423 (1969).

4. К н я з и х и н Ю., Изв. АН ЭССР, Физ. Матем., 30, № 2, 140-146 (1981).

5. К ан торович Л., В., Акилов Г. П., Функциональный анализ, М., «Наука», 1979.

Институт астрофизики и физики атмосферы Академии наук Эстонской ССР

Поступила в редакцию 9/III 1981

\section{J. KNJAZIHHIN}

\section{ARVUTUSVIGADE MOJUST ATMOSFÄARI NEELAVATE KOMPONENTIDE MÄARAMISE POORDULESANDE LAHENDILE}

On uuritud atmosfääri neelavate komponentide määramise pöördülesande vea hinnangut (viga on põhjustatud mitmekordselt hajunud kiirguse mõju lihtsustatud käsitlusest) ja tuldud järeldusele, et tehteid lihtsustavad eeldused ei môjuta oluliselt lahendi täpsust optiliselt õhukese atmosfääri korral.

\section{J. KNJAZIHHIN}

\section{ON THE EFFECT OF CALCULATING ERRORS IN SOLVING THE INVERSE PROBLEM OF ASTROPHYSICS FOR ABSORBING COMPONENTS OF THE ATMOSPHERE}

Measurements aboard the orbital station «Salyut-4», in 1975, gave a number of vertical brightness profiles of the atmosphere in the near-infrared region over different geographical areas for different heights of the Sun. The present measurement gives a possibility to solve the inversion problem of these profiles for various components of the atmosphere.

The author has already presented a method for the inversion of water vapour concentration $\left[{ }^{4}\right]$. This paper continues the work. It deals with the estimation of errors made in solving the inversion of the absorbing components of the atmosphere. The errors appear due to inaccuracies in the calculation of multiple scattering. For this purpose a set $S(\tilde{\eta})$ was constructed, including the exact solution. The set was obtained with the help of monotonic sequences (9). The method developed was applied for the estimation of errors made in the inversion of vertical brightness profiles for water vapour concentrations in the transfer model ignoring multiple scattered radiation. 